\title{
ON THE FORMATION OF HOT DQ WHITE DWARFS
}

\author{
L. G. Althaud ${ }^{1,4}$, E. García-Berro ${ }^{2,3}$, A. H. Córsico ${ }^{1,4}$, M. M. Miller Bertolami ${ }^{1,5}$, and A. D. Romero ${ }^{1,5}$ \\ ${ }^{1}$ Facultad de Ciencias Astronómicas y Geofísicas, Universidad Nacional de La Plata, Paseo del Bosque s/n, (1900) La Plata, Argentina; althaus@fcaglp.unlp.edu.ar \\ ${ }^{2}$ Departament de Física Aplicada, Escola Politècnica Superior de Castelldefels, Universitat Politècnica de Catalunya, Av. del Canal Olímpic, s/n, 08860 \\ Castelldefels, Spain \\ ${ }^{3}$ Institut d’Estudis Espacials de Catalunya, c/Gran Capità 2-4, 08034 Barcelona, Spain \\ Received 2008 December 9; accepted 2009 January 8; published 2009 February 11
}

\begin{abstract}
We present the first full evolutionary calculations aimed at exploring the origin of hot DQ white dwarfs. These calculations consistently cover the whole evolution from the born-again stage to the white dwarf cooling track. Our calculations provide strong support for the diffusive/convective mixing picture for the formation of hot DQs. We find that the hot DQ stage is a short-lived stage and that the range of effective temperatures where hot DQ stars are found can be accounted for by different masses of residual helium and/or different initial stellar masses. In the frame of this scenario, a correlation between the effective temperature and the surface carbon abundance in DQs should be expected, with the largest carbon abundances expected in the hottest DQs. From our calculations, we suggest that most of the hot DQs could be the cooler descendants of some PG 1159 stars characterized by He-rich envelopes markedly smaller than those predicted by the standard theory of stellar evolution. At least for one hot DQ, the high-gravity white dwarf SDSS J142625.70+575218.4, an evolutionary link between this star and the massive PG 1159 star H1504+65, is plausible.
\end{abstract}

Key words: stars: evolution - stars: individual (H1504+65, SDSS J142625.70+575218.4) - stars: interiors - white dwarfs

\section{INTRODUCTION}

White dwarf stars constitute the end-point of stellar evolution for the overwhelming majority of stars. In fact, more than $97 \%$ of all stars in our Galaxy are expected to end their lives as white dwarfs. Thus, the present white dwarf population contains information about the history of our Galaxy and has also potential applications as reliable cosmic clocks to infer the age of a wide variety of stellar populations; see Winget \& Kepler (2008) and Fontaine \& Brassard (2008) for recent reviews.

Traditionally, white dwarfs have been classified into two distinct families according to the main chemical constituents characterizing their surfaces: those with a H-dominated atmosphere - the DA spectral type-which comprises about $85 \%$ of known white dwarfs, and those with a He-rich surface composition - the non-DA white dwarfs - which represent the rest of the population. It is accepted that most non-DA white dwarfs are the direct descendants of PG 1159 stars-see Unglaub \& Bues (2000) and Althaus et al. (2005) and references therein-which are hot stars with H-deficient and He-, C-, and O-rich surface layers (Werner \& Herwig 2006). In fact, PG 1159 stars constitute a transition stage between the post-asymptotic giant branch (AGB) stars and most of the H-deficient white dwarfs. A significant fraction of PG 1159 stars are thought to be formed as a result of a born-again episode, that is, a very late thermal pulse (VLTP) experienced by a hot white dwarf during its early cooling phase (Iben et al. 1983; Herwig et. al. 1999). During the VLTP, the He flash-driven convection zone reaches the H-rich envelope of the star and, consequently, most of the hydrogen is violently burned (Herwig et al. 1999; Miller Bertolami et al. 2006). The star is then forced to evolve rapidly back to the AGB and finally as the central star of a planetary nebula at high effective temperatures. Ultimately, gravitationally induced

\footnotetext{
${ }^{4}$ Member of the Carrera del Investigador Científico y Tecnológico, CONICET (IALP), Argentina.

5 Fellow of CONICET.
}

diffusion acting during the early stage of evolution leads to the formation of a pure He envelope, giving rise to the DO and DB spectral types; see, for instance, Althaus et al. (2005) and references therein.

The recent discovery of a new lukewarm population of white dwarfs with C-rich atmospheres-known as hot DQs (Dufour et al. 2007, 2008a) - has sparked the attention of researchers since it has raised the possibility of the existence of a new evolutionary channel of formation. Dufour et al. (2008a) have reported that nine hot white dwarfs identified in the Fourth Data Release of the Sloan Digital Sky Survey (SDSS) are characterized by atmospheres dominated by carbon. The existence of these new white dwarfs - all of them found in a narrow effective temperature strip (between $\approx 18,000 \mathrm{~K}$ and $24,000 \mathrm{~K}$ ) - poses a challenge to the stellar evolution theory, which cannot adequately explain their origin. As proposed by Dufour et al. (2008a), the hot DQ population could be related to the very hot $(\approx 200,000 \mathrm{~K})$ and massive $\left(0.83 M_{\odot}\right)$ member of the PG 1159 family, the enigmatic star H1504+65, for which a post born-again origin is not discarded (Althaus et al. 2009). Interestingly enough. H1504+65 was the only known star with no traces of either $\mathrm{H}$ or He until the discovery of the hot DQ white dwarfs. Dufour et al. (2008a) have outlined an evolutionary scenario in which undetected traces of He remaining in the $\mathrm{C}$ - and $\mathrm{O}$-rich outer layers of H1504+65 would diffuse upward leading to a He-rich white dwarf. In this picture, a C-rich atmosphere should eventually emerge as the result of convective mixing at smaller effective temperatures. In this Letter, we present the first quantitative assessment of such an evolutionary scenario. With the help of full evolutionary models that consistently cover the evolution from the born-again stage to the cooling track, we present strong theoretical evidence supporting a diffusive/convective mixing picture such as that proposed by Dufour et al. (2008a) for the formation of hot DQs and the existence of an evolutionary link between these stars and the PG 1159 stars, including H1504+65. 


\section{INPUT PHYSICS AND EVOLUTIONARY SEQUENCES}

The calculations presented in this work have been done using the LPCODE stellar evolutionary code employed in our previous study of the formation of $\mathrm{H}$-deficient post-AGB stars via a born-again episode (Althaus et al. 2005; Miller Bertolami \& Althaus 2006). The code is specifically designed to compute the formation and evolution of white dwarf stars. In LPCODE, special emphasis is given to the treatment of the changes of the chemical abundances, including diffusive overshooting and non-instantaneous mixing, which are of primary importance in the calculation of the thermal pulses and born-again stage that lead to the formation of PG 1159 stars. We have considered the following main physical ingredients. Neutrino emission rates for pair, photo, and bremsstrahlung processes were taken from Itoh et al. (1996). For plasma processes we included the treatment presented in Haft et al. (1994). The OPAL radiative opacities (Iglesias \& Rogers 1996), including C- and O-rich compositions, were adopted. The conductive opacities are from Cassisi et al. (2007). This prescription covers the entire regime where electron conduction is relevant. In the low-density regime, we employed an updated version of the equation of state of Magni \& Mazzitelli (1979). For the high-density regime, we use the equation of state of Segretain et al. (1994), which accounts for all the important contributions in the solid and liquid phases. We have also considered the gravitational settling and chemical diffusion of He, $\mathrm{C}$, and $\mathrm{O}$ during the whole evolution. Our treatment of time-dependent diffusion is based on the multicomponent gas treatment presented in Burgers (1969). Diffusion velocities are evaluated at each evolutionary time step. Finally, convection is treated within the formalism of the mixing length theory as given by the ML2 parameterization (Tassoul et al. 1990).

The initial stellar models needed to start our evolutionary sequences correspond to realistic PG 1159 stellar configurations derived from the full evolution of their progenitor stars (Miller Bertolami \& Althaus 2006). We have considered sequences with stellar masses of 0.87 and $0.585 M_{\odot}$. The chemical stratification of our initial models consists of a CO core, which is the result of core He burning in prior stages, surrounded by a He-, C-. and O-rich envelope, in agreement with what is observed in PG 1159 stars. To explore the possibility that hot DQ white dwarfs can be the result of mixing events between the He-rich envelope and the underlying $\mathrm{C}$-rich regions, we have considered small masses of residual $\mathrm{He}$ with fractional masses in the range $10^{-8} \leqslant M_{\mathrm{He}} / M_{\mathrm{WD}} \leqslant 2 \times 10^{-7}$. The sequence with $0.87 M_{\odot}$ was specifically computed to explore the evolutionary connection between H1504+65 and hot DQ white dwarfs. For this stellar mass, the He content of $2 \times 10^{-7} M_{\mathrm{WD}}$ corresponds to the maximum He content expected in $\mathrm{H} 1504+65$ if we assume a post-born-again origin for this star (Miller Bertolami $\&$ Althaus 2006). For this sequence we assume an outer layer rich in $\mathrm{C}$ and $\mathrm{O}$, with trace abundances of $\mathrm{He}$ reaching deeper layers. The evolutionary calculations have been computed from $T_{\text {eff }}=100,000 \mathrm{~K}$ down to the domain of effective temperatures typical of hot DQs.

\section{EVOLUTIONARY RESULTS}

Figure 1 illustrates the main result of our calculations about the diffusive/convective mixing picture for the formation of hot DQs. We show the results for the $0.87 M_{\circ}$ sequence with $M_{\mathrm{He}}=10^{-8} M_{\mathrm{Wl}}$. As previously mentioned, this stellar mass is similar to that of H1504+65. During the hot phase of white dwarf evolution. the chemical abundance distribution of the

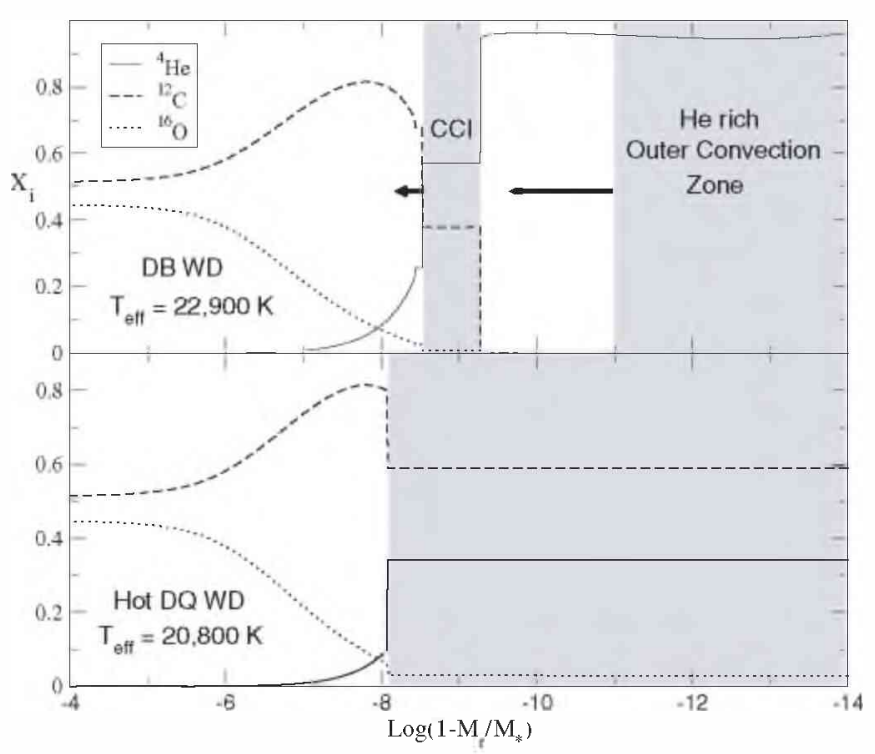

Figure 1. Abundance distribution of ${ }^{4} \mathrm{He},{ }^{12} \mathrm{C}$, and ${ }^{16} \mathrm{O}$ as a function of the outer mass fraction at two selected effective temperatures for the $0.87 M_{\odot}$ white dwarf with $M_{\mathrm{He}}=10^{-8} M_{\mathrm{WD}}$. Gray areas denote convectively unstable zones. The inward-growing outer convection zone (upper panel) merges with the underlying convective $\mathrm{C}$-rich intershell (CCI), leading to the formation of a white dwarf with a $\mathrm{C}$ atmosphere-a hot $\mathrm{DQ}$ - at about $T_{\text {eff }}=20,800 \mathrm{~K}$ (bottom panel).

envelope of PG 1159 stars is strongly modified by element diffusion. Gravitational settling causes He to float to the surface and heavier elements to sink. In our models when the white dwarf has cooled down to $T_{\text {eff }} \approx 23,000 \mathrm{~K}$, gravitationally induced diffusion has led to the development of a pure $\mathrm{He}$ envelope of nearly $7 \times 10^{-10} M_{\mathrm{WD}}$, plus an extended tail toward deeper layers (upper panel in Figure 1). Note that at this stage of the evolution, an inward-growing outer He convection zone is present. This convective region is due to He recombination.

The large values of the radiative opacity of the C-enriched layers below the pure He envelope produce a convective intershell zone stretching downward, which increases the carbon abundance there. As the evolution proceeds, the base of this convective region digs deeper into C-rich layers, thus further increasing the abundance of carbon in the intershell. The existence of this convective intershell is a key aspect to understand the formation of hot DQs. It is worth mentioning that the less massive the He envelope, the larger the size of the convective intershell and the larger the $\mathrm{C}$ enrichment of the intershell. When $T_{\text {eff }}=21,000 \mathrm{~K}$, the outer He convection zone reaches the underlying convective intershell, now substantially enriched in $\mathrm{C}$. The resulting mixing process between the two convective regions gives rise to the formation of a white dwarf with outer layers rich in He and $\mathrm{C}$ (and small traces of $\mathrm{O}$ ), which is a hot DQ (the bottom panel in Figure 1).

Although the evolution described above is qualitatively similar for sequences with different He contents, the effective temperature at which mixing occurs and the surface chemistry after mixing depend quite sensitively upon the exact value of the residual He mass and on the stellar mass. In fact, the surface $\mathrm{C}$ enrichment of the emerging hot DQ depends on the size of the convective intershell, which is larger for the case of a smaller He envelope. These results are summarized in Table 1 which, in addition to the stellar mass and He content, lists the effective temperature and surface abundances of $\mathrm{He}, \mathrm{C}$, and $\mathrm{O}$ by the time the outer convection zone merges with the convective intershell. It is clear that the range of effective temperature where 
Table 1

Main Characteristics of the White Dwarf Evolutionary Sequences by the Time the Outer Convection Zone Leads to the Formation of C-Rich Outer Layers

\begin{tabular}{lccccc}
\hline \hline$M_{\mathrm{WD}} / M_{\odot}$ & $M_{\mathrm{He}} / M_{\mathrm{WD}}$ & $T_{\text {eff }}(\mathrm{K})$ & ${ }^{4} \mathrm{He}$ & ${ }^{12} \mathrm{C}$ & ${ }^{16} \mathrm{O}$ \\
\hline 0.870 & $10^{-8}$ & 20.700 & 0.36 & 0.58 & 0.03 \\
0.870 & $2 \times 10^{-7}$ & 17.300 & 0.63 & 0.31 & 0.02 \\
0.585 & $10^{-8}$ & 21.100 & 0.17 & 0.77 & 0.03 \\
0.585 & $2 \times 10^{-7}$ & 19.500 & 0.74 & 0.22 & 0.003 \\
\hline
\end{tabular}

hot DQs are found, as well as the observed surface chemistry, can be naturally accounted for by assuming different masses of the residual He content with which white dwarfs enter their cooling track and/or different white dwarf masses. Finally, it is worth mentioning that the residual He content required for this scenario to work is much larger than the value of $10^{-15} M_{\odot}$ suggested by Dufour et al. (2008a), and consequently easier to justify by prior evolution.

\section{AN EVOLUTIONARY CONNECTION BETWEEN H1504+65 AND HOT DQ WHITE DWARFS?}

Our $0.87 M_{\odot}$ sequence is of immediate relevance for the discussion of an evolutionary link between the massive H1504+65 star and hot DQs. Note from Table 1 that for this sequence and for a He content between $M_{\mathrm{He}} \approx 10^{-8}$ and $10^{-7} M_{\mathrm{WD}}$, the formation of a DQ white dwarf is expected to occur at nearly $T_{\text {eff }}=20,000 \mathrm{~K}$, and the predicted surface composition is expected to be $\mathrm{He}$ and $\mathrm{C}$ in roughly equal fractions. This is in very good agreement with the observational values derived for the high-gravity hot DQ white dwarf SDSS J142625.70+575218.4. for which Dufour et al. (2008a) obtain an effective temperature of nearly $T_{\text {eff }}=20,000 \mathrm{~K}$ and a He- and C-rich surface composition of $\log (\mathrm{C} / \mathrm{He})=0$.

Is the amount of He required in this case consistent with the residual He mass that could be expected in a star like H1504+65? As mentioned, H1504+65 is the hottest and most massive known PG 1159 star. Its atmosphere is dominated by $\mathrm{C}$ and $\mathrm{O}$ plus traces of heavier elements- $X_{\mathrm{C}} \simeq 0.48, X_{\mathrm{O}} \simeq 0.48, X_{\mathrm{Ne}} \simeq 0.02$. $X_{\mathrm{Mg}} \simeq 0.02$ (Werner et al. 2004). Recently, Althaus et al. (2009) have explored different evolutionary scenarios for its formation and they do not discard a post-born-again origin for this star as a result of non-stationary mass loss after the VLTP. From detailed simulations of the born-again episode for massive remnants (Miller Bertolami \& Althaus 2006), it is found that even in the case in which most of the He-rich envelope is eroded during the Sakurai stage, a non-negligible mass of He remains in relatively deep layers. This is illustrated in Figure 2 for the $0.87 M_{\odot}$ postborn-again sequence. Note that the total amount of residual He below the layer having a He abundance of 0.01 -the detection limit of He in H1504+65 (Werner et al. 2004)-amounts to $M_{\mathrm{He}}=2 \times 10^{-7} M_{\odot}$, which can be smaller if He in the surface layers of H1504+65 exists with abundances much lower than the detection limit. This is on the order of magnitude of the He mass required by the diffusive/convective mixing picture described here to explain the observational characteristics of SDSS J142625.70+575218.4. Thus, we have a consistent picture that provides theoretical support for an evolutionary connection between $\mathrm{H} 1504+65$ and this hot DQ white dwarf.

\section{CONCLUSIONS}

We have presented full evolutionary calculations that provide strong support for a diffusive/convective mixing picture such as that proposed by Dufour et al. (2008a) to explain the origin

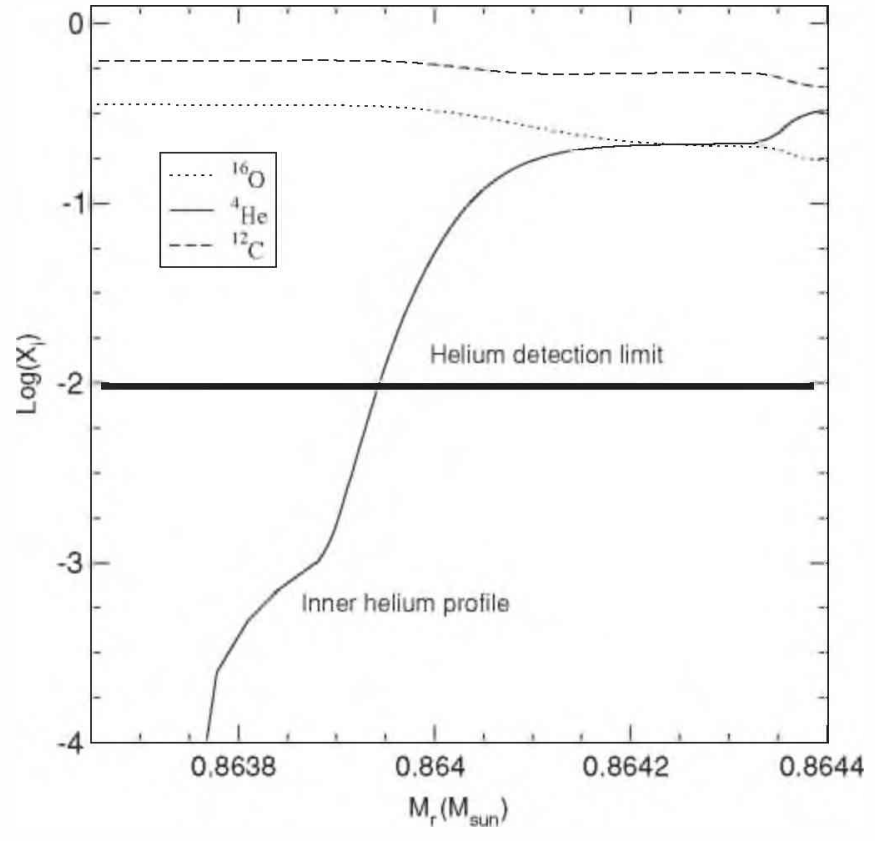

Figure 2. Internal abundance distribution of ${ }^{4} \mathrm{He},{ }^{12} \mathrm{C}$, and ${ }^{16} \mathrm{O}$ for a selected model of our post-born-again $0.87 M_{\curvearrowright}$ sequence. The mass coordinate ranges from 0.86365 to $0.8644 M_{\odot}$. Note that a non-negligible mass of residual $\mathrm{He}$, of about $\sim 2 \times 10^{-7} M_{\odot}$, is expected below the layer having a He abundance of 0.01, the He detection limit in H1504+65 (thick line).

of hot DQ white dwarfs. We have shown that mixing between the outer He convection zone with the underlying convective carbon intershell gives rise to white dwarf structures with the appropriate effective temperatures and surface compositions inferred from the observation of hot DQs. With the help of detailed born-again simulations for massive remnants, the scenario described in this work provides the first theoretical evidence for an evolutionary link between the hydrogen- and He-deficient PG 1159 star H1504+65 with the high-gravity DQ white dwarf SDSS J142625.70+575218.4, a connection that can be traced back to strong mass-loss episodes after the VLTP.

For the intermediate-gravity DQ white dwarfs we find that the scenario predicts the formation of C-dominated envelopes for residual He contents on the order of $M_{\mathrm{He}}=10^{-8} M_{\mathrm{WD}}$ or smaller (see Table 1). As the residual He content is increased, the formation of hot DQs at lower effective temperatures and with smaller $\mathrm{C}$ abundances at their surfaces is expected. According to this scenario, we should thus expect a correlation between the effective temperature and the surface $\mathrm{C}$ abundance, with the largest $\mathrm{C}$ abundances expected in the hottest DQs. This trend appears to be in agreement with the observational inferences (Dufour et al. 2008a). Numerical difficulties prevent us from following the further evolution of our sequences beyond the merger of the two convection zones. However, we can make some estimations. For the evolutionary models shown in Figure 1 we note that the convection zone just after the formation of the hot DQ reaches as deep as $10^{-8} M_{\odot}$. At the base of the convective zone the diffusion timescale is much shorter (ranging from $\tau_{\mathrm{dif}} \approx 10^{5}$ to $10^{6} \mathrm{yr}$ ) than the cooling timescale $\left(\tau_{\text {cool }}=T_{\mathrm{c}} / \bar{T}_{\mathrm{c}} \approx 10^{8} \mathrm{yr}\right)$. Hence, we expect that the hot DQ stage is indeed a short-lived stage in the evolution of a white dwarf. This being the case, the range of effective temperature where the hot DQs are found could be accounted for by assuming different masses of the residual He with which white dwarfs enter the cooling track and/or different stellar masses. However, it cannot be discarded that additional mixing episodes could develop with 
further cooling. Additional calculations would be needed to test this possibility.

Finally, we note that the origin of intermediate-gravity DQ white dwarfs is unclear. As suggested by Dufour et al. (2008a), the presence of these hot DQs could be indicating the existence of a new evolutionary scenario for the formation of white dwarfs. In view of the evolutionary connection between H1504+65 and the high-gravity SDSS J142625.70+575218.4, we suggest that the hot DQ white dwarf population of intermediate gravity could also be the descendants of some PG 1159 stars. For this to be possible in the frame of the evolutionary picture described here, some PG 1159 stars should be characterized by He contents orders of magnitude smaller than predicted by the standard theory of stellar evolution. This possibility is not discarded by the recent theoretical findings of Althaus et al. (2008), which suggest that a thin He-rich envelope appears to be needed to solve the longstanding discrepancy between the observed (Costa \& Kepler 2008) and theoretical (Córsico et al. 2008) rates of period change of the pulsating star PG 1159035, the prototype of the PG 1159 stars. This being the true course of events, we should face the problem of explaining the coexistence of PG 1159 stars characterized by markedly different He contents. In this sense, the presence of a companion star in a PG 1159 star (Nagel et al. 2006) may be indicating another channel of formation of these stars. In closing, it is worth noting that at least for one hot DQ, a strong magnetic field has been detected (Dufour et al. 2008b). As discussed by these authors, the presence of this magnetic field might affect the convection zones significantly, thus altering the predictions of the diffusive/convective mixing scenario described here.

Part of this work was supported by the MEC grant AYA0508013-C03-01, by the European Union FEDER funds, by the AGAUR, by AGENCIA through the Programa de Modern- ización Tecnológica BID 1728/OC-AR, and by PIP 6521 grant from CONICET

\section{REFERENCES}

Althaus, L. G., Córsico, A. H., Miller Bertolami, M. M., García-Berro, E., \& Kepler, S. O. 2008, ApJ, 677, L35

Althaus, L. G., Córsico, A. H., Torres, S., \& García-Berro, E. 2009, A\&A, in press (arXiv:0811.4154)

Althaus, L. G., Serenelli, A. M., Panei, J. A., Córsico, A. H., García-Berro, E., \& Scoccola, C. G. 2005, A\&A, 435, 631

Burgers, J. M. 1969, in Flow Equations for Composite Gases (New York: Academic)

Cassisi, S., Pothekin, A.Y., Pietrinferni, A., Catelan, M., \& Salaris, M. 2007, ApJ, 661, 1094

Corsico, A. H. , Althaus, L. G., Kepler, S. O., Costa, J. E. S., \& Miller Bertolami, M. M. 2008, A\&A, 478, 869

Costa, J. E. S., \& Kepler, S. O. 2008, A\&A, 489, 1225

Dufour, P., Fontaine, G., Liebert, J., Schmidt, G.D., \& Behara, N. 2008a, ApJ, 683,978

Dufour, P., Fontaine, G., Liebert, J., Williams, K., \& Lai, D. K. 2008b, ApJ, $683, \mathrm{~L} 167$

Dufour, P., Liebert, J., Fontaine, G., \& Behara, N. 2007, Nature, 450, 522

Fontaine, G., \& Brassard, P. 2008, PASP, 120, 1043

Haft, M., Raffelt, G., \& Weiss, A. 1994, ApJ, 425, 222

Herwig, F., Blocker, T., Langer, N., \& Driebe, T. 1999, A\&A, 349, L5

Iben, I. Jr, Kaler, J. B., Truran, J. W., \& Renzini, A. 1983, ApJ, 264, 605

Iglesias, C. A., \& Rogers, F. J. 1996, ApJ, 464, 943

Itoh, N.. Hayashi, H., Nishikawa, A., \& Kohyama, Y. 1996. ApJS, 102. 41

Magni, G., \& Mazzitelli, I. 1979, A\&A, 72, 134

Miller Bertolami, M. M., \& Althaus, L. G. 2006, A\&A, 454, 845

Miller Bertolami, M. M., Althaus, L. G., Serenelli, A. M., \& Panei, J. A. 2006, A\&A, 449, 313

Nagel, et al. 2006, A\&A, 448, L25

Segretain, L., Chabrier, G., Hernanz, M., García-Berro, E., \& Isern, J. 1994, ApJ, 434,641

Tassoul, M., Fontaine, G., \& Winget, D. 1990, ApJS, 72, 335

Unglaub, K., \& Bues, I. 2000, A\&A, 359, 1042

Werner, K., \& Herwig, F. 2006, PASP, 118,183

Werner, K., Rauch, T., Barstow, M. A., \& Kruk, J. W. 2004, A\&A, 421, 1169

Winget, D. E., \& Kepler, S. O. 2008, ARA\&A, 46, 157 